\title{
Astrovirology: Expanding the Search for Life
}

White Paper for the Planetary Science and Astrobiology Decadal Survey 2023-2032

July 15,2020

Primary author: Gareth Trubl, (602)317-8208, Lawrence Livermore National Lab, trubl1@1lnl.gov

Co-authors: Kenneth Stedman (Portland State University), Kathryn Bywaters (Honeybee Robotics), Penelope J. Boston (NASA Ames Research Center), Jason T. Kaelber (Rutgers University), Simon Roux (Lawrence Berkeley National Lab), Joanne B. Emerson (University of California, Davis), Mya Breitbart (University of South Florida), John Yin (University of Wisconsin-Madison), Aleksandar Janjic (Technische Universität München), Pacifica Sommers (University of Colorado Boulder), Eduardo Rodríguez-Román (Instituto Venezolano de Investigaciones Científicas)

\section{Co-signatories:}

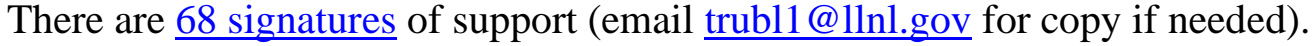

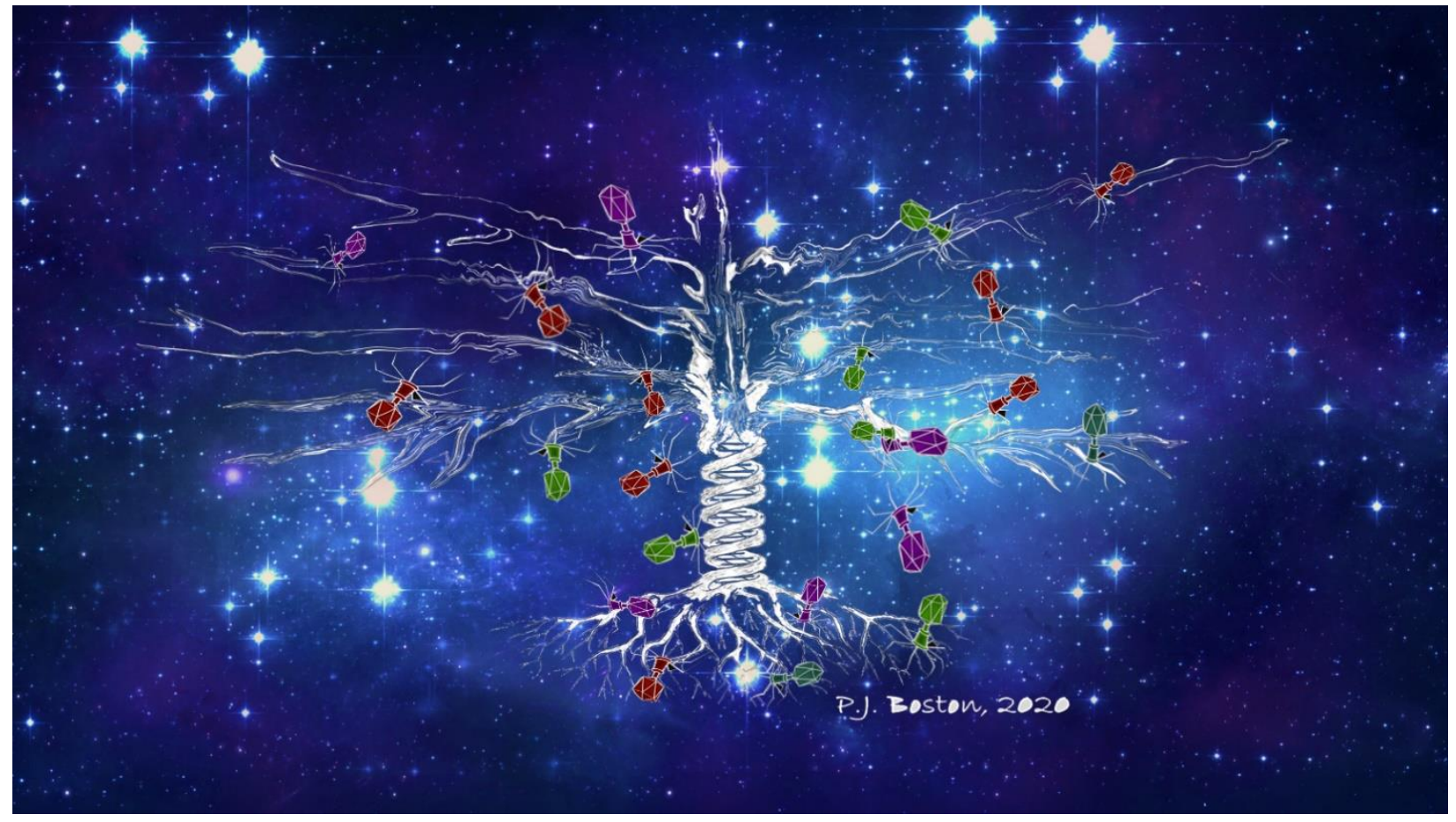




\section{Background for Astrovirology}

Viruses are the most numerically abundant biological entities on Earth [1]. Viruses, essentially replicators of information molecules and agents of change, could play a critical role in other potential biospheres and life detection missions to other planetary bodies [2]. However, there are major knowledge gaps about the virosphere such as the role viruses play in biogeochemical cycles, the origin(s) of viruses, and the involvement of viruses in the evolution, distribution, and persistence of life on Earth and beyond. By studying replicators that span all known types of nucleic acid, we expand our experimental and theoretical toolbox for detecting and understanding life on Earth and beyond. It is only by filling in these knowledge gaps that we will obtain an inclusive assessment of how to distinguish and detect life on other planetary surfaces.

Astrovirology will contribute to addressing key scientific questions in planetary science, space biology and astrobiology, such as:

1. How did life and viruses originate and what was the mechanism \& timeline of evolution into cellular and complex multicellular organisms?

2. What environmental conditions and processes are most conducive to the preservation and propagation of information molecules or molecular signatures of life?

3. What role(s) could acellular informational molecules play in potential biospheres elsewhere?

4. Can the detection of informational molecules beyond Earth be interpreted as a sign of life?

5. Can understanding how natural versus artificial environments drive changes in the virome lead to a better understanding of viral regulation and controls within an ecosystem?

6. Can biosignature isotopic variation in cellular organisms be explained by viral infection?

Viruses meet most, but not all, proposed definitions of life [3], although these definitional considerations remain controversial [4-5]. What is unambiguous is that viruses are a fundamental part of the living world. Therefore, although we refer to "viral life" in this white paper for reasons of clarity, the same conclusions would be drawn if viruses are considered nonliving.

\section{Addressing these key scientific questions}

\section{How did life and viruses originate and what was the mechanism \& timeline of evolution into cellular and complex multicellular organisms?}

Viruses have played and continue to play a critical role in the evolution of life on Earth. Precursors of virus-like entities may even mark the beginning of life itself, making the virosphere likely at least as old and significant as the actual biosphere [6-7]. Viral signatures may be pivotal in the search for life elsewhere and in understanding evolutionary mechanics of other biospheres. Theoretical models predict that, depending on information-transfer properties of the system, parasitic replicators will emerge anywhere in the universe that life evolves [8-10]. Even viruses fall prey to parasitic replicators in the form of defective virus genomes that multiply at the expense of fully intact virus genomes [11]. The RNA-world hypothesis characterizes the origin of life with self-replicating RNA, followed by ribonucleoproteins that later evolved into DNA and larger proteins. Viruses and virus-like replicators are the only known extant biological entities that contain all types of genomes, including single-stranded and double-stranded RNA and DNA or mixtures thereof. RNA viruses serve as models for how RNA and ribonucleoproteins could have propagated via simple self-replicating RNA structures and ribozyme activity [12-17]. Therefore, viruses likely helped support the transition from early RNA-world to the current DNA-world [1819]. Further, viruses played a role in the origins of the placenta [20] and of the mitochondrion [21], 
and they may also have been critical in the evolution of multicellularity and of eukaryotes [2225]. While uncovering the history of viruses is a key to understanding the origin of life on Earth, an additional benefit is the development of new technologies for characterizing the diversity and history of non-terran life. For instance, while all cells conserve some genomic features such as ribosomal RNA, alternate methods are required to detect and classify viruses from genomic data [26-27]. Non-Earth life may lack nucleic acids: comparative analysis of 3D atomic structure is a non-genomic method being used to interrogate virus origins [28]. Understanding the role of viruses in the origin of life on Earth and their persistence in extreme environments therefore plays an essential role in the search for life elsewhere, one of the three core contexts of NASA's first strategic objective 1.1: Understand the Sun, Earth, Solar System and Universe.

\section{Future directions}

a) Investigate evolutionary relationships between RNA viruses and ribozymes to evaluate the potential role of viruses in the RNA-World, and transition to the DNA World.

b) Create tools and methods to make inferences about life in the Archaean Eon through comparative analysis of extant viral life.

c) Determine the origin of viruses on Earth and the role viruses played in the emergence of life by reconstructing ancient events.

d) Develop phylogenies that consider all biological entities to better constrain the shared and/or divergent evolutionary histories of viruses and cellular organisms.

\section{What environmental conditions and processes are most conducive to the preservation and propagation of information molecules or molecular signatures of life?}

Environmental factors drive the ability of viruses to persist and maintain their integrity for extended periods of time. For instance, viruses adsorb to clays in a manner that protects them from inactivation, enabling them to persist in soils for longer periods of time in the absence of their hosts [29]. Diverse viruses have also been shown to persist in inhospitable conditions such as droplets suspended in the atmosphere [30], permafrost [31-32] or other icy environments such as ice cores or cryoconite holes [33-35], hot springs [36-37], chemically harsh conditions [38], deep sea sediments [39-40], and virus remnants are found in most cellular genomes [41]. Viruses have evolved multiple mechanisms to survive these conditions and in doing so, protect their host, for instance viruses have been shown to confer heat tolerance to their host [42] or carry genes for sporulation $[32 ; 43]$. Additionally, viruses have the potential to aid in preservation of biological signatures. Viruses infecting microbial mats can potentially cause or expedite their fossilization into a stromatolite by acting as a nucleation site for organomineralization, altering microbial host metabolism for carbonate precipitation, or increasing the production of microbial exopolymeric substances [44-45]. Virus particles in the environment can be preserved via silicification and have been reactivated (i.e. infectious) when the silica is removed [37]. Further, upon thawing, viruses preserved in frozen permafrost for $\sim 30,000$ years were able to infect modern day versions of their hosts [46]. Overall, these different studies clearly outline viruses and specifically viral capsids as key structures for preservation and propagation of biological information, especially in extreme environments. In this context, a better understanding of the key environmental and molecular factors driving this long-term persistence will inform future searches for virus-like elements outside of Earth.

Future directions

a) Develop long-term environmental monitoring strategies for viruses. 
b) Characterize viruses that inhabit extreme environments on Earth as these can be used as analogs of celestial bodies and early life on Earth.

c) Examine environments and conditions that enhance viral preservation (e.g. arid, oxygen content, humidity, or amber).

d) Combine techniques to improve detection and characterization of viruses (e.g. stable isotope probing-metagenomics).

\section{What role(s) could acellular informational molecules play in potential biospheres elsewhere?}

Our understanding of viruses as major players in many of Earth's biogeochemical cycles has been entirely reshaped by the advent of metagenomic approaches that enabled the study of uncultivated viruses [47-49]. For example, marine cyanobacteria are responsible for about $25 \%$ of the oxygen we breathe and at any moment about half of them are infected by viruses that can either lyse these organisms preventing oxygen production or increase the efficiency of their oxygen production [50]. Marine virology has been intensely studied, highlighting the potential for worlds with liquid oceans, such as Enceladus or Europa, to contain informational molecules like viruses among their organic compounds [51]. Other work demonstrating the activity of viruses in frozen soils [31] illustrates the potential for viruses to be important in biogeochemical processes of terrestrial worlds as well, such as Mars. Terrestrial worlds, or at least partly rocky bodies such as comets, may host ice-lidded cryoconite holes in their polar ice caps that could result in the presence of liquid water to support active life [52]. We can even consider locations like Venus, which could harbor iron and sulfur driven metabolism, that viruses have been identified to augment [48; 53-55], on its surface or within its thick atmosphere where clouds are made of sulfuric acid droplets.

The widespread and frequent detection of genes used by viruses to hijack the metabolism of their host cell(s) and manipulate them to produce progeny viruses strengthens the need to for a conceptual shift, where infected cells are now considered as virocells [56-57]. Such an infection changes a microbe's metabolic outputs, impacting the composition and quantity of their mostly unexplored biosignatures. It is now feasible to computationally integrate how encoded functions in virus genomes interact with material and energy resources of their hosts to predict the timing and levels of virus growth [58]. Combined experimental and in silico studies of virus growth on bacteria hosts have shown how the physiological state of the host cell can be reflected in the timing and level of virus production, where virus production is intimately linked to the availability of resources for protein synthesis. Since nutrient availability in the host's environment impacts its ability to produce virus, the productivity of virus infection can provide a readout of the metabolic demands of the living host [59]. Further computational modeling enables a compelling case to be made that virus evolve to optimally use the finite metabolic energy resources of their host cells [60]. Similar virus-host interactions would likely be at play in other systems and it is thus critical to expand our understanding of these interactions on Earth to enable the detection and identification of such phenomenon in other biospheres.

\section{Future directions}

a) Obtain a better understanding of how viruses hijack their host cellular machinery.

b) Further explore the broad range of virus-host interactions and dynamics in nature.

c) Quantitatively estimate the role and impact(s) of virocell metabolism in Earth's different biogeochemical cycles.

\section{Can the detection of informational molecules beyond Earth be interpreted as a sign of life?}


Potential exists for the development of flight instruments that can detect either virus particles or sequences. For example, solid-state nanopore-based biosensors are being investigated by various groups for space flight and have proven capable of evaluating different biomarkers (i.e. DNA/RNA, proteins, and whole viral particles - spanning from a few nanometers to over 100 nanometers in size). Solid-state nanopores can also discriminate between viral particles by monitoring the change in electrical current as viruses pass through an electrically biased pore or used to measure the mass-density of viruses [61-64] as well as particle-size distributions in virus populations [65]. Current technologies that are being configured for future missions can readily be applied to detect and characterize viruses. For example, nanoscale secondary ion mass spectroscopy (NanoSIMS) can perform in situ quantitative trace sample analysis with exceptional sensitivity and spatial resolution. NanoSIMS is currently being evaluated by the Network for Life Detection to help discern between abiotic and biological signatures based on how elemental and isotopic patterns are different in cellular organisms versus from minerals and other precipitates. The NanoSIMS instrument has recently demonstrated the capability to not only detect viruses, but also to map their elemental and isotopic distributions in complex communities and in mineralized samples, suggesting viruses play a role in organomineralization and may important for how life gets preserved in the geological record [44]. An atomic force microscope was used during the Phoenix Mars Lander to investigate Martian soils [66] and in February 2020, a scanning electron microscope was added to the International Space Station (ISS) [67]. These technologies complement spectroscopic methods by providing orthogonal evidence for viral and nonviral life [668]. Given their ability to persist in extreme environments, the multiple approaches available to detect them, and their dependence on cellular life, viruses might be key signs of life beyond Earth.

Independent of in situ investigations, the identification of virus particles and genomes can be applied to planetary protection and sample-return missions. Recently, the use of propidium monoazide on clean room samples removed 'relic' DNA allowing the detection of viruses and microbes [69]. It must therefore be ensured in advance that methods of environmental virology will also be applied to instruments used for life detection and on returned samples [7].

\section{Future directions}

a) Develop flight-ready technology for more efficient and accurate detection and characterization of virus genomes.

b) Investigate innovative technologies for automated detection and identification of virus and virus-like particles.

c) Apply such technologies in Earth analog systems to explore their prospects and limitations.

d) Incorporate viruses in standard operating procedures for return flight missions and in examination of samples from return missions.

\section{Can understanding how natural versus artificial environments drive changes in the virome lead to a better understanding of viral regulation and controls within an ecosystem?}

Space flight stresses biological systems, including immune systems that fight virus infections, but these effects are not fully understood [70]. The reaction of viruses to microgravity is poorly known, for not only human viruses, but also for viruses infecting components of the human microbiome and proposed life support organisms, such as plants or cyanobacteria. After 6-12 months in space, astronauts had significant changes in their microbiome that led to rashes and hypersensitivity episodes, warranting further investigation of the effects of long-term exposure from the space environment on humans and their microbes and viruses [71]. It has been shown that astronauts, 
subjected to the stresses of space flight, experience reactivation of latent viruses, such as herpes simplex virus [72-74]. Clearly, viruses will be critical factors in astronaut health and safety. To enable long-duration crewed missions beyond low Earth orbit, it is essential to understand the influence of the space environment on viruses, their hosts, and their interactions.

\section{Future directions}

a) Compare human and plant viromes and the behavior of viruses in artificial environments.

b) Investigate viromes in built environments relevant to space, including the ISS.

c) Explore virus-host dynamics in space for human- and plant-associated microbiomes.

d) Assess triggers that cause latent infections to become virulent.

\section{Can biosignature isotopic variation in cellular organisms be explained by viral infection?}

Microbial life preferentially incorporates the lighter isotope available for each compound, but the exact signatures of this process depend on the organism(s) performing a given metabolic process, how many enzymatic reactions take place in relevant metabolic pathways, and how the enzyme(s) work. For example, fractionation factors are different for denitrification via fungi relative to bacteria [75], or for methanogenesis depending on the initial substrate and the organisms involved [76-78]. When a virus infects a microbial host, it redirects its metabolism, thus impacting these isotopic values. This could be more dramatic if a virus encodes an auxiliary metabolic gene that has a different fractionation factor than the host version of that gene. For example, kinetic measurements of virus and host versions of the same enzyme revealed that the virus enzyme had a significantly lower $\mathrm{kcat} / \mathrm{Km}$ value than the host enzyme [79]. These viral influences can lead to large variations in isotopic signatures, leading to uncertainty when distinguishing between abiotic and biological processes and in the utility of a biosignature, or potentially providing a virus biosignature [80]. Isotope fractionation thus has the potential to reveal fundamental characteristics of biosphere metabolism, including the impact and contribution of viruses.

Isotopic fractionation can be applied to the geologic record on Earth as well as on Mars to infer characteristics of the metabolisms of past biospheres, and changes over time [81]. The isotope history of marine ecosystems throughout Earth history is well studied [82-83]. The isotopic composition of icy environments through time on Earth is less well characterized but may provide analog environments to other planetary bodies such as Mars [84]. Distinguishing viral influences on both present isotopic signatures and on their variation over multiple times scales may therefore provide a reference for interpreting isotopic biosignatures of past life on other worlds.

\section{Future directions}

a) Improve the understanding of viral impacts on biosignatures for major Earth biogeochemical cycles, including the magnitude of their impact.

b) Investigate the existence of generalizable biosignatures associated with viral metabolism reprogramming to reduce uncertainty associated with biosignatures for life detection.

\section{Virological aspects of other programs}

Above, we have outlined ways in which focused astrovirological investigation can advance the goals of planetary science. However, across the diverse disciplines of astrobiology, several classes of investigations cannot afford to neglect viruses. Most of the diversity of life on earth is comprised of viruses, whether diversity is defined by number of species, type of genetic information, number of individuals [1;85-87], absence of any universally present gene, mode of replication, or number of unique (i.e. non-homologous) genes [88]. Viruses should be explicitly considered in organism- 
level astrobiological investigations. For instance, metagenomic surveys that collect mainly ribosomal RNA data are of limited utility to astrobiology because they can reflect only a subset of terran biological diversity (although other studies of the ribosome remain important to understanding how terran life emerged). The ability to detect virus-like organisms should be a point of assessment for instruments and missions to directly detect extraterrestrial organisms, and Mars sample return materials should be examined for the presence of viruses [7]. When organisms are used as model systems, viruses should be adequately represented. This consideration will reduce biases and increase utility of diverse astrobiological studies.

"Whether viruses are alive or not may be a moot question, but if a virion (or a virus-like particle) were to be unequivocally detected in an extraterrestrial sample, very few people would claim that this would not be evidence for life-wherever that sample was from”. - Berliner et al. (2018)

\section{Conclusion}

For accurate and effective pursuits in the field of Astrobiology it is critical to understand how life on Earth functions, as it is our only sample to interrogate. Viruses are key contributors to Earth's ecosystems, yet there is much yet unknown regarding their influence on cellular life, role in evolutionary history, and their fundamental physical interactions with the Earth system, and many basic ecological factors such as persistence and decay under various scenarios. This white paper addresses key questions that need to be examined to advance the field of astrobiology by incorporating viruses: the most abundant yet poorly understood biological entities on planet Earth and perhaps beyond.

\section{References}

[1] Mushegian, A.R. 2020. J. of Bacteriology, 202(9). [2] Berliner, A. J. et al. 2018. Astrobiology, 18(2), pp.207-223. [3] Domingo, E. 2020. Virus as Populations, p.1. [4] Moreira, D. and LópezGarcía, P. 2009. Nature Reviews Microbiology, 7(4), pp.306-311. [5] Koonin, E.V. and Starokadomskyy, P. 2016. Stud Hist Philos Biol Biomed Sci, 59, pp.125-134. [6] Moelling, K. and Broecker, F. 2019. Geosciences, 9(5), p.241. [7] Janjic, A. (2018). Astrobiology 18:1611-1614. [8] Vlok, M. Gibbs, A.J. and Suttle, C.A. 2019. Viruses, 11(3), p.299. [9] Eigen, M. 1971. Naturwissenschaften, 58(10), pp.465-523. [10] Bresch, C. Niesert, U. and Harnasch, D. 1980. J. of theoretical biology, 85(3), pp.399-405. [11] Perrault, J. 1981. Springer, Berlin, Heidelberg. [12] Tyler, K. L. 2008. Lancet Infect Dis 8(4):224. [13] Matsumura, S. Kun, Á. et al. 2016. Science, 354(6317), pp.1293-1296. [14] Koonin, E.V. Senkevich, T.G. and Dolja, V.V. 2006. Biology direct, 1(1), p.29. [15] Landweber, L.F. Simon, P.J. and Wagner, T.A. 1998. BioScience, 48(2), pp.94-103. [16] Weinberg, C.E. Weinberg, Z. and Hammann, C. 2019. Nucleic acids research, 47(18), pp.9480-9494. [17] Durzyńska, J. and Goździcka-Józefiak, A. 2015. Virology j., 12(1), p.169. [18] Forterre, P. 2006. PNAS, 103(10), pp.3669-3674. [19] Diemer, G. S. \& Stedman, K. M. 2012. Biol Direct 7(1):13. [20] Mi, S. et al. 2000. Nature, 403(6771), pp.785-789. [21] Shutt, T.E. and Gray, M.W. 2006. Trends in Genetics, 22(2), pp.90-95. [22] Forterre, P. and Gaïa, M. 2016. Current Opinion in Microbiology, 31, pp.44-49. [23] Lee, G. et al. 2018. PNAS, 115(49), pp.12465-12470. [24] Guglielmini, J. et al. 2019. PNAS, 116(39), pp.19585-19592. [25] Iranzo, J. et al. 2014. Cell Cycle, 13(19), pp.3083-3088. [26] Roux, S. et al. 2015. Elife, 4, p.e08490. [27] Tisza, M.J. et al. 2020. Elife, 9, p.e51971. [28] Krupovic, M. and Koonin, E.V. 2017. PNAS, 114(12), pp.E2401-E2410. [29] Stotzky G 1989. McGraw-Hill, New York. [30] Reche, I. et al. 2018. The ISME j., 12(4), pp.1154-1162. [31] Emerson, J.B. et al.2018. Nature microbiology, 3(8), pp.870-880. [32] Trubl, G. et al. 2018. MSystems, 3(5). [33] Wells, L.E. and Deming, J.W. 2006. Env. Microbiology, 8(6), pp.1115-1121. [34] Zhong, Z.P, et al. 2020. Msystems, 5(3). [35] 
Sommers, P. et al. 2020. Viruses, 11(11), p.1022. [36] Laidler, J.R. and Stedman, K.M. 2010. Astrobiology, 10(6), pp.569-576. [37] Laidler, J.R. et al. 2013. J. of virology, 87(24), pp.1392713929. [38] Gupta, K. Lee, Y. \& Yin, J. (1995). J. of Molecular Evolution, 41(2), 113-114. [39] Engelhardt, T. Orsi, W.D. and Jørgensen, B.B. 2015. Environmental microbiology reports, 7(6), pp.868-873. [40] Cai, L. et al. 2019. The ISME j., 13(7), pp.1857-1864. [41] Bannert, N. and Kurth, R. 2004. PNAS, 101(suppl 2), pp.14572-14579. [42] Márquez, L.M. et al. 2007. Science, 315(5811), pp.513-515. [43] Van Goethem, M.W. et al. 2019. Mbio, 10(6). [44] Pacton, M. et al. 2014. Nature Comm 5(1):1-9. [45] White III, R.A. Visscher, P.T. and Burns, B.P. 2020. Trends in Microbiology. [46] Legendre, M. 2014. PNAS 111(11):4274-4279. [47] Kristensen, D.M. et al. 2010. Trends in microbiology, 18(1), pp.11-19. [48] Roux, S. et al. 2016. Nature 537(7622):689693. [49] Roux, S. et al. 2019. Nature biotechnology, 37(1), pp.29-37. [50] Sieradzki, E. T. et al. 2019. Nature Comm 10(1):1-9. [51] Postberg, Frank, et al. 2018. Nature 558.7711 (2018): 564568. [52] Zawierucha, Krzysztof, Marta Ostrowska, and Małgorzata Kolicka. 2017. Contemporary Trends in Geoscience 6. [53] Bonnain, C. Breitbart, M. and Buck, K.N. 2016. Frontiers in marine science, 3, p.82. [54] Anantharaman, K. et al. 2014. Science, 344(6185), pp.757-760. [55] Martins, P.D. et al. 2018. Microbiome, 6(1),pp.1-17. [56] Forterre, P. 2011. Comptes Rendus Chimie, 14(4), pp.392-399. [57] Forterre, P. 2013. ISME J 7(2):233-236. [58] Yin, J. and Redovich, J. 2018. Microbiology and Molecular Biology Reviews, 82(2). [59] You, L. Suthers, P. F. \& Yin, J. (2002). J. of bacteriology, 184(7), 1888-1894. [60] Kim, H. \& Yin, J. 2004. Biotechnology and Bioengineering, 88(2), 148-156. [61] Arjmandi, N. et al. 2014. Analyt Chem 86(10):4637-4641. [62] Zhou, K. et al. 2011. J Amer Chem Soc 133(6):1618-1621. [63] McMullen, A. et al. 2014. Nature Comm 5:4171. [64] Wu, H. et al. 2016. Analyt Chem 88(4):2502-2510. [65] Akpinar, F. \& Yin, J. (2015). J. of Virological Methods, 218, 71-76. [66] Pike, W.T. et al. 2011. Geophysical Research Letters, 38(24). [67] Own, C.S. et al. 2018. LPI, (2083), p.2756. [68] Zhang, Y. Hung, T. Song, J. and He, J. 2013. Science China Life Sciences, 56(5), pp.421-430. [69] Weinmaier, T. et al. 2015. Microbiome 3, 62. 10.1186/s40168-015-0129-y. [70] Crucian, B.E. et al. 2008. AVIAT SPACE ENVIR MD, 79(9), pp.835-843. [71] Voorhies, A.A. et al. 2019. Scientific reports, 9(1), pp.1-17. [72] Rooney, B. V. et al. 2019. Front Microbiol 10 16. [73] Stowe, R. P. et al. 2000. Neuroimmunomodulation 8(2):51-58. [74] Mehta, S. K. et al. 2004. J Med Virol 72(1):174-179. [75] Ostrom, N.E. and Ostrom, P.H. 2017. Biogeochemistry, 132(3), pp.359-372. [76] Whiticar, M.J. 1999. Chemical Geology, 161(1-3), pp.291-314. [77] Hornibrook, E.R. Longstaffe, F.J. and Fyfe, W.S. 2000. Geochimica et Cosmochimica Acta, 64(6), pp.1013-1027. [78] Penning, H. et al. 2006. AEM, 72(8), pp.5648-5652. [79] Thompson, L.R. et al. 2011. PNAS, 108(39), pp.E757E764. [80] Schwieterman, E.W. Kiang, N.Y. Parenteau, M.N. Harman, C.E. DasSarma, S. Fisher, T.M. Arney, G.N. Hartnett, H.E. Reinhard, C.T. Olson, S.L. and Meadows, V.S. 2018. Astrobiology, 18(6), pp.663-708. [81] Johnson, B. et al. (2017). Nature Communications, 8(1):1316. [82] Krissansen-Totton, J. Buick, R. and Catling, D. C. 2018. American J. of Science, 315(4):275-316. [83] Zerkle, A. and Mikhail, S. 2017. Geobiology, 15:343-352. [84] Havig, J. R. and Hamilton, T. L. 2019. Geochimica et Cosmochimica Acta, 247:220-242. [85] Breitbart, M. 2002. PNAS 99, no. 22 (2002): 14250-14255. [86] Roossinck, M. J. 2012. Annual review of genetics, 46, 359-369. [87] Paez-Espino, D. et al. 2016. Nature, 536(7617), 425-430. [88] Koonin, E.V. and Wolf, Y.I. 2012. Frontiers in cellular and infection microbiology, 2, p.119.

The work conducted by the LLNL was contributed under the auspices of the US Department of Energy under Contract DE-AC52-07NA27344, and at the Lawrence Berkeley National Laboratory through Contract No. DE-AC02-05CH11231. 